\title{
Magnetization Reversal in Ferromagnetic Nanorings of Fourfold Symmetries
}

\author{
Andrea Ehrmann ${ }^{1}$ and Tomasz Blachowicz ${ }^{2}$ \\ ${ }^{1}$ Faculty of Engineering and Mathematics, Bielefeld University of Applied Sciences, Interaktion 1, 33619 Bielefeld, Germany \\ ${ }^{2}$ Institute of Physics, Center for Science and Education, Silesian University of Technology, Ul. Konarskiego 22B, 44-100 Gliwice, Poland
}

Correspondence should be addressed to Andrea Ehrmann; andrea.ehrmann@fh-bielefeld.de

Received 31 January 2017; Revised 4 September 2017; Accepted 18 September 2017; Published 16 October 2017

Academic Editor: Jamal Berakdar

Copyright (C) 2017 Andrea Ehrmann and Tomasz Blachowicz. This is an open access article distributed under the Creative Commons Attribution License, which permits unrestricted use, distribution, and reproduction in any medium, provided the original work is properly cited.

\begin{abstract}
Magnetic nanoparticles offer a broad spectrum of magnetization reversal processes and respective magnetic states, such as onion, horseshoe, or vortex states as well as various states including domain walls. These states can be correlated with stable intermediate states at remanence, enabling new quaternary memory devices storing two bits in one particle. The stability of these intermediated states was tested with respect to shape modifications, variations in the anisotropy axes, and rotations and fluctuations of the external magnetic field. In our micromagnetic simulations, 6 different stable intermediate states were observed at vanishing magnetic field in addition to the remanence state. The angular region of approx. $5^{\circ}-12^{\circ}$ between nanoring and external magnetic field was identified as being most stable with respect to all modifications, with an onion state as technologically best accessible intermediate state to create quaternary memory devices.
\end{abstract}

\section{Introduction}

Magnetic nanoparticles have gained the interest of several research groups due to their unique magnetic properties, enabling their possible application in magnetic storage media, sensors, MRAMs, spin valves, and so on [1-6]. In magnetic nanoparticles, shape anisotropy is significantly stronger than magnetocrystalline and magnetoelastic anisotropy so that the magnetic properties can be changed by modifications of the particle shape [7]. This is why magnetic nanodots and nanorings were shown to enable formation of a broad variety of magnetic states, such as onion or horseshoe, flux-closed vortex states, and domain wall states [8-15].

Particularly "square rings" of different shapes and materials have shown interesting magnetic properties. In several situations, hysteresis loops with steps on both sides occur in theoretical and experimental investigations, allowing creating "quaternary" memory systems with four stable states at vanishing external magnetic field and thus the possibility of storing two bits in one position [16]. On the other hand, experimental investigation of these structures has revealed undesired modifications of the original rings' shapes in the lithography process, resulting in modified magnetization reversal processes $[17,18]$. This experience underlines the necessity of examining the influence of such shape modifications to allow creation of nanorings with reliable magnetic properties.

In this paper, phase diagrams of magnetic states are presented for "ideal" and "real" square rings, varying the magnetocrystalline anisotropy axes between a random state, as in a typical sputtered sample, and two epitaxial states, as they could be gained by molecular beam epitaxy (MBE) [19] or self-assembly [20], for a complete in-plane rotation of the nanoring with respect to the external field.

\section{Materials and Methods}

For modelling, the micromagnetic simulator OOMMF (Object Oriented Micromagnetic Framework) [21] was used which is based on finite differences (discretizing space into small cuboids) and the Landau-Lifshitz-Gilbert (LLG) equation of motion [22]. 


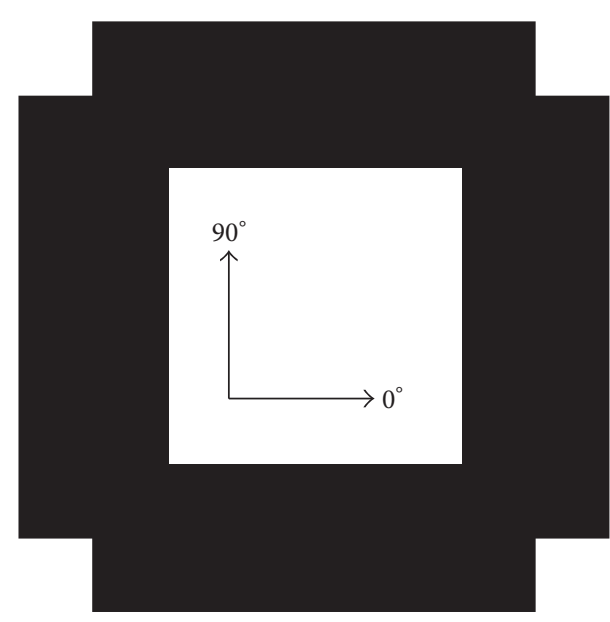

(a)

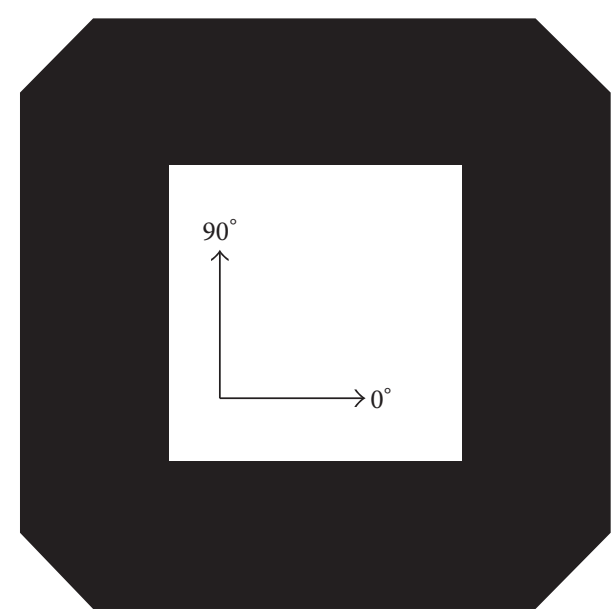

(b)

FIGURE 1: Ring shapes used for simulations with square (a) and diagonal corner cuts (b).

Nanostructures of two different geometries were chosen for simulation (Figure 1): one square ring with "ideal" square corner cuts which has shown to result in intermediate stable states at remanence [16] and one ring with "realistic" diagonal corner cuts which was gained in former lithographic processes $[17,18]$. Lateral dimensions were chosen as $200 \mathrm{~nm}$ diameter and $5 \mathrm{~nm}$ height, similar to real nanorings under experimental investigation $[17,18]$.

As material, iron $(\mathrm{Fe})$ was chosen which showed ideal properties to reach the aforementioned intermediates states [23]. The chosen parameters were as follows: saturation magnetization $M_{S}=1700 \cdot 10^{3} \mathrm{~A} / \mathrm{m}$, exchange constant $A=$ $21 \cdot 10^{-12} \mathrm{~J} / \mathrm{m}$, anisotropy constant $K_{1}=48 \cdot 10^{3} \mathrm{~J} / \mathrm{m}^{3}$, Gilbert damping constant $\alpha=0.1$, and mesh diameter $d=5 \mathrm{~nm}$. These values are consistent with typical literature values [24]. The damping constant is slightly increased, compared with values measured in $\mathrm{Fe}$ nanostructures [25], to avoid oscillations which would not occur in real experiments with relatively slow field sweeps.

To avoid statistical variations which would superpose the effects due to interactions between the neighboring nanorings, the influence of thermal fluctuations of temperatures above zero was ignored. It can be assumed that any temperature above zero will further decrease the field regions in which all nanorings show the same behavior.

The anisotropy was on the one hand assumed to be uniform on the macroscale, that is, having randomly distributed axes in each cell, to create a sputtered nanoring with polycrystalline structure. In other simulations, the anisotropy axes were fixed at $0^{\circ} / 90^{\circ}$ as well as at $\pm 45^{\circ}$ to create both extremal cases of epitaxially grown nanorings.

The external magnetic field was applied parallel to the sample plane. The sample orientation was varied between $0^{\circ}$ (external magnetic field parallel to the $x$-axis of the sample/to one side of the nanoring) and $45^{\circ}$ in steps of $5^{\circ}$. Maximum external fields between $\pm 0.2 \mathrm{~T}$ and $\pm 0.3 \mathrm{~T}$ were used, depending on the angle, to ensure reaching magnetization saturation.
Additionally, minor loops were simulated to investigate the possibility of creating additional stable magnetic states at vanishing external magnetic field.

\section{Results and Discussion}

Figure 2 depicts a typical hysteresis loop, simulated in the angular region of $1^{\circ}-15^{\circ}$. While the (positive) saturated state is an onion state, magnetization reversal occurs via a horseshoe state, another anion, and a second horseshoe until finally the next onion state is reached which is the (negative) saturated state. These magnetization states are shown in Figure 3. While the intermediate onion state occurs nearly always in this angular region, the horseshoe states may vanish, depending on the angle, geometry, and anisotropy (epitaxial or sputtered) of the nanoring.

While these states are also visible in the "realistic" shape with diagonal corner cuts, other states occur exclusively in the "ideal" nanorings with square corner cuts with randomly distributed anisotropy axes, that is, in a "sputtered" nanoring. These states are depicted in Figure 4. In all these states, domain walls block the formation of a vortex state.

In Figure 5, phase diagrams of the minor loops via onion states are shown. At first glance, both situations, square or diagonal corner cuts, look similar. Between $1^{\circ}$ and approx. $17^{\circ}$, minor loops via onion states are possible for which the connection fields $H_{\text {Connect }}$ and the minor coercive fields $H_{\text {Minor }}$ are given. For small angles, the field distances between $H_{\text {Coerc }}$ and $H_{\text {Connect }}$ are relatively large, allowing for switching into the intermediate onion state by stopping the field sweep within a large field area to go back to zero magnetic field. This is helpful for technical applications in storage devices, and so on. Nevertheless, there is also a significant difference in the angular area around $30^{\circ}$ visible where the coercive fields of the nanorings with square corner cuts show a maximum while those of the nanorings with diagonal corner cuts increase continuously. 


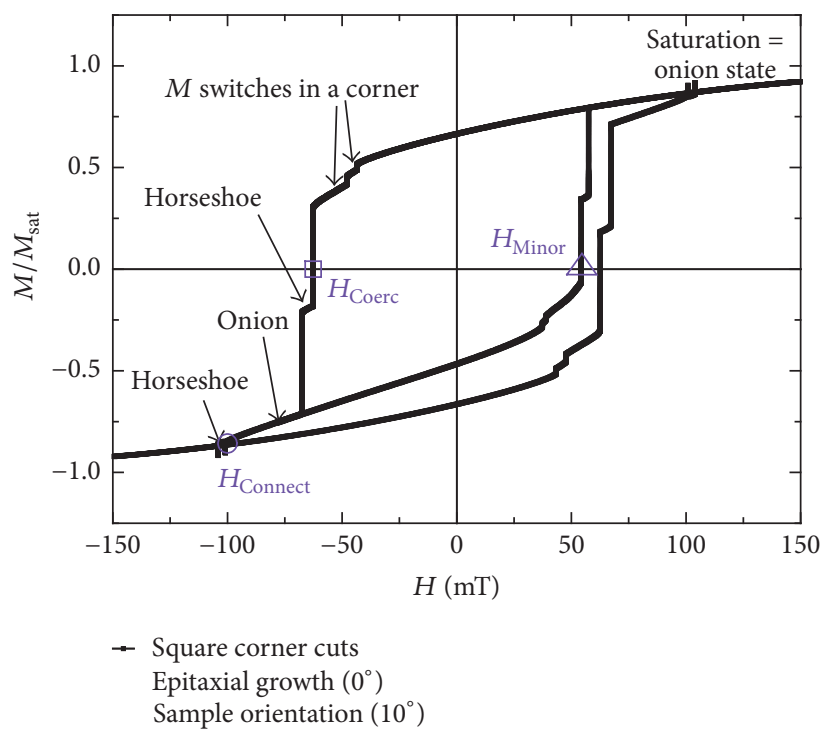

FIGURE 2: Sample hysteresis loop with the main magnetization states identified as onion and horseshoe states. Lilac marks depict the coercive field $H_{\text {Coerc }}$ as well as the fields defined as $H_{\text {Connect }}$ and $H_{\text {Minor }}$.

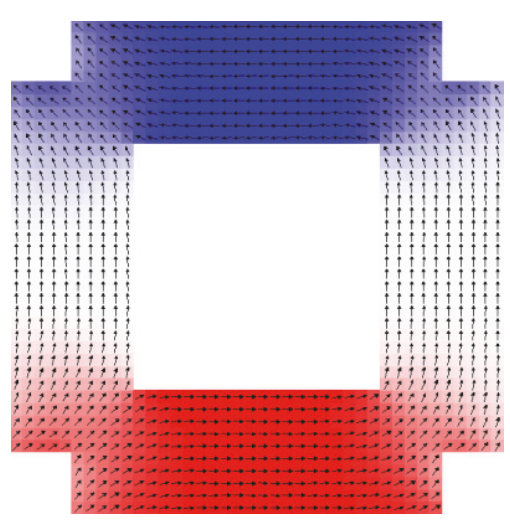

(a)

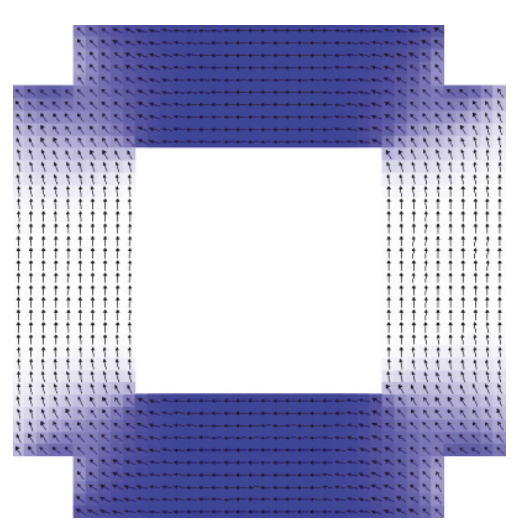

(b)

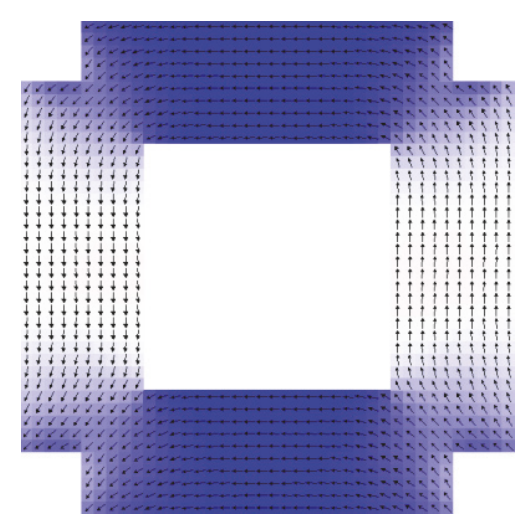

(c)

FIgURE 3: Possible magnetic states for not-too-large angles: horseshoe 1 (a), onion (b), and horseshoe 2 (c).

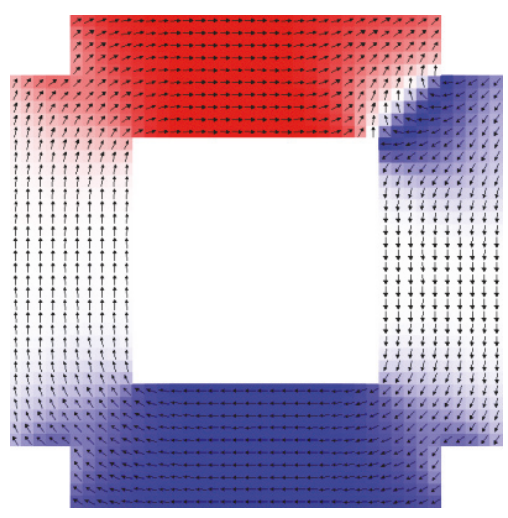

(a)

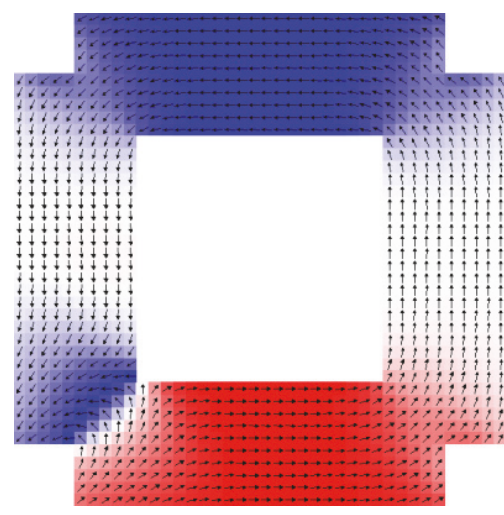

(b)

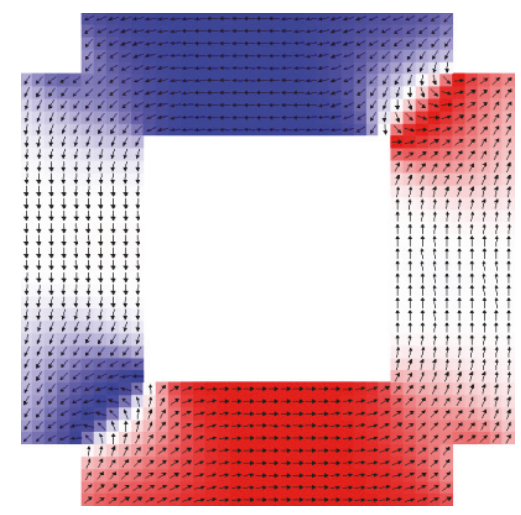

(c)

FIGURE 4: Possible magnetic states for larger angles, simulated in a nanoring with square corner cuts and randomly distributed anisotropy axes: one $360^{\circ}$ domain wall (a), one $360^{\circ}$ domain wall (b), and two $360^{\circ}$ domain walls (c). 

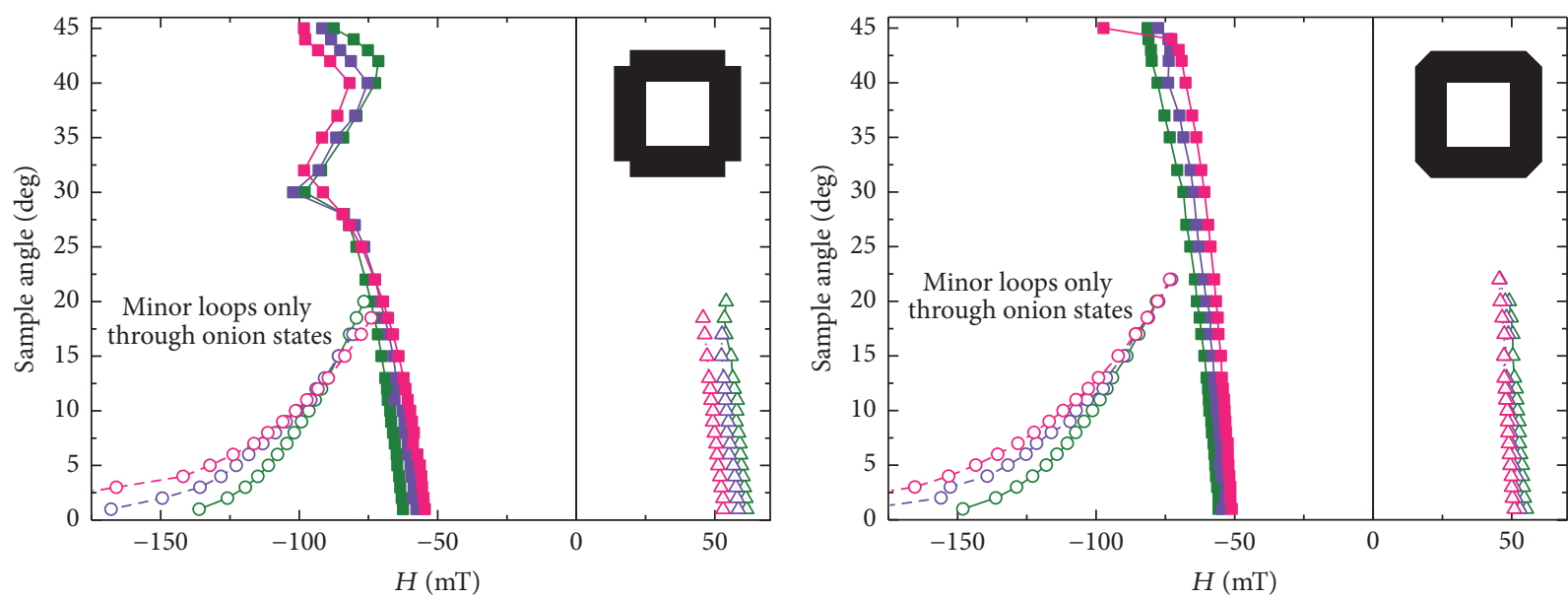

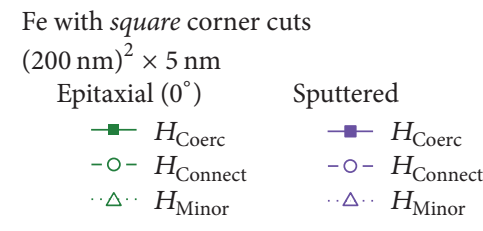

(a)

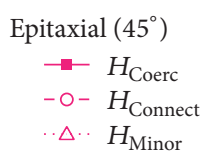

A. $H_{\text {Minor }}$

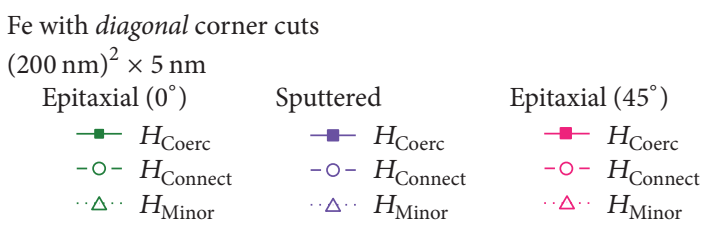

(b)

FIGURE 5: Phase diagrams of minor loops via onion states, simulated for nanorings with square corner cuts (a) and diagonal corner cuts (b). The fields $H_{\text {Coerc }}, H_{\text {Connect }}$, and $H_{\text {Minor }}$ are explained in Figure 2.

To evaluate the reason for this difference as well as the occurrence of other possible magnetic states, Figure 6 depicts the complete phase diagrams for the nanorings with square corner cuts, separated with respect to the three anisotropy situations. For both epitaxial nanorings, only onion states are visible; additional steps in the hysteresis loop are correlated with instable modifications of the magnetization distribution in corners which are reversed by small field changes.

In case of a random distribution of the anisotropy axes, that is, a sputtered sample, most of the smaller angles up to approx. $17^{\circ}$ reverse magnetization in the aforementioned steps, that is, from positive saturated onion via first horseshoe, intermediate onion, and second horseshoe to negative saturated onion. Nevertheless, for some angles this process works without horseshoe states. In this special nanoring, additional domain wall states may occur for larger angles (cf. Figure 4). While the state with two $360^{\circ}$ domain walls is only assumed for $45^{\circ}$, for angles between $17^{\circ}$ and $43^{\circ}$ one of the states with one $360^{\circ}$ domain wall may be taken. While this happens more often in some angles and less often in others, the influence of arbitrary anisotropy axes distribution can change this behavior at any time.

It should be mentioned that the sputtered sample shows more deviations from a clear correlation between sample angle and coercive fields due to the arbitrary distribution of anisotropy axes. This effect results in small variations of the simulated values for repeated simulations.

In Figure 7, the phase diagrams of the nanorings with diagonal corner cuts are shown. Firstly it is visible that no domain wall states occur. On the other hand, in all three anisotropy geometries, the phase diagrams look similar, with the angular region below approx. $20^{\circ}$ reversing magnetization via the above describe route, that is, from positive saturated onion via first horseshoe, intermediate onion, and second horseshoe to negative saturated onion, while there are no intermediate states available for higher angles.

In general, it can be stated that the nanoring with diagonal corner cuts shows a more reliable, less arbitrary spectrum of magnetization reversal mechanisms. Due to their statistic character, the domain wall states in the "sputtered" sample with square corner cuts are only of academic interest. From the technical point of view, those angular regions are best suited which

(i) avoid instabilities, for example, arising from symmetry axes, such as $0^{\circ}$, or from changes in the magnetization reversal processes, such as around $17^{\circ}-20^{\circ}$;

(ii) are similar for patent shape and diagonal corner cuts;

(iii) are similar for sputtered and both epitaxial samples;

(iv) are similar for neighboring angles;

(v) have large differences between $H_{\text {Coerc }}$ and $H_{\text {Connect }}$, since in this way they can simply be reached and are thus well reproducible;

(vi) have large differences between $H_{\text {Coerc }}$ and $H_{\text {Minor }}$, since in this way they are robust against small field deviations due to crosstalk from neighboring nanorings. 

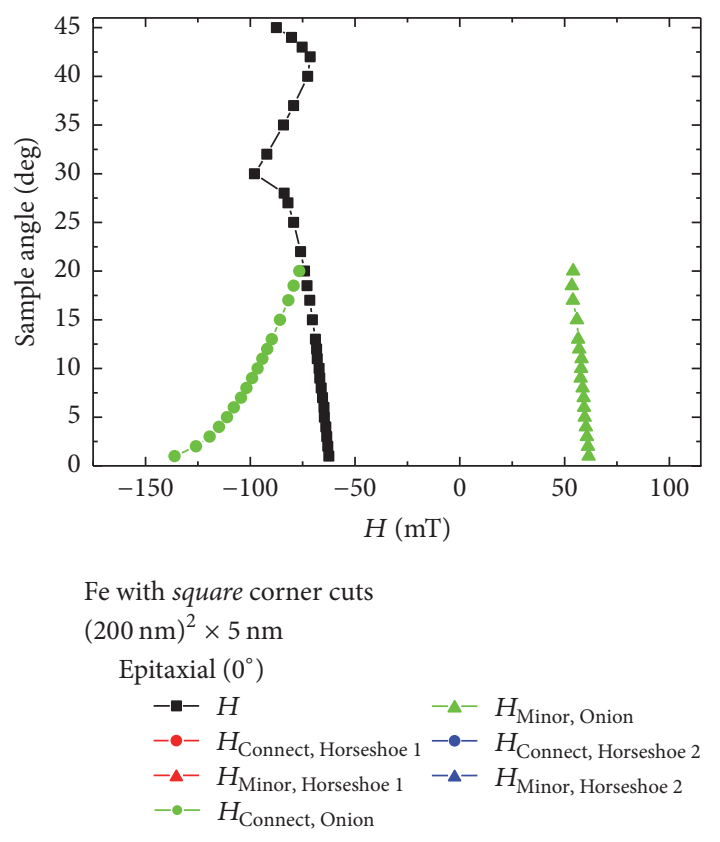

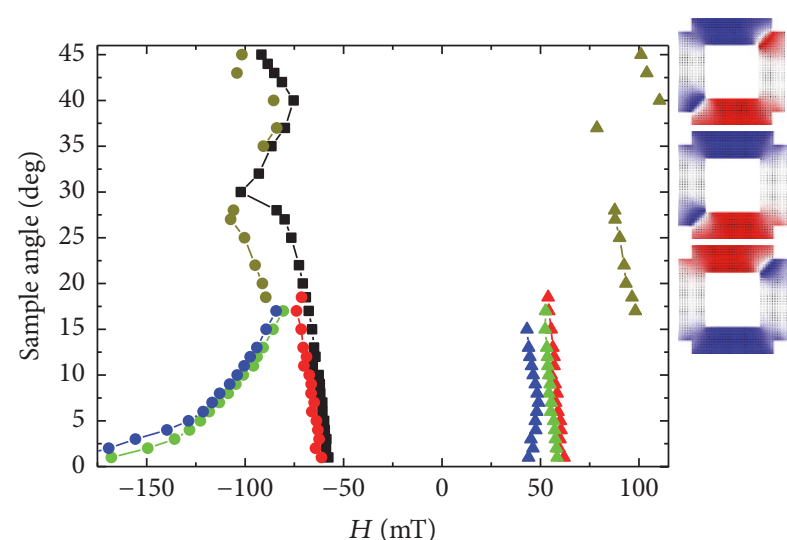

Fe with square corner cuts $(200 \mathrm{~nm})^{2} \times 5 \mathrm{~nm}$ Sputtered

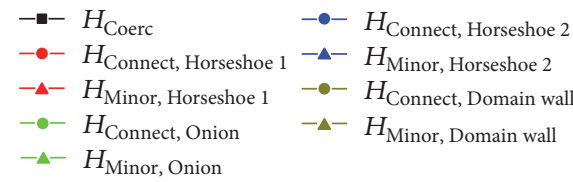

(b)

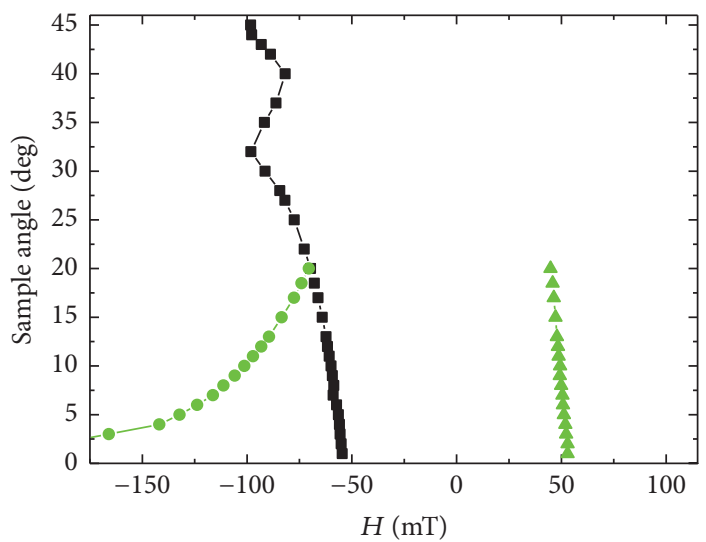

Fe with square corner cuts $(200 \mathrm{~nm})^{2} \times 5 \mathrm{~nm}$ Epitaxial $\left(45^{\circ}\right)$

$-\square-H_{\text {Coerc }} \quad-\Delta-H_{\text {Minor, Onion }}$

$-\bullet H_{\text {Connect, Horseshoe 1 }}-\bullet-H_{\text {Connect, Horseshoe } 2}$

$-\mathbf{-}-H_{\text {Minor, Horseshoe 1 }}-\mathbf{\Delta}-H_{\text {Minor, Horseshoe } 2}$

- $H_{\text {Connect, Onion }}$

(c)

Figure 6: Phase diagrams simulated for nanorings with square corner cuts, assuming epitaxial anisotropy orientation along $0^{\circ}$ (a), $45^{\circ}$ (c), and random anisotropy axes (b). The colors of the texts correspond to those in Figure 5.

This means that a sputtered sample in an orientation around $5^{\circ}-10^{\circ}$ should be preferred in technological applications, such as quaternary magnetic storage devices.

\section{Conclusions}

To conclude, we have given an overview of different magnetization reversal processes and magnetic states occurring in square nanorings grown by sputtering or epitaxially from iron. Using micromagnetic simulations, we have shown the variety of magnetic states which can be reached in such nanostructures, depending on the exact shape, anisotropy orientation, and external magnetic field direction. While some processes exhibited a statistical nature and could not be repeated, an angular region around $5^{\circ}-10^{\circ}$ between external field and nanoring side has proven to reverse magnetization 


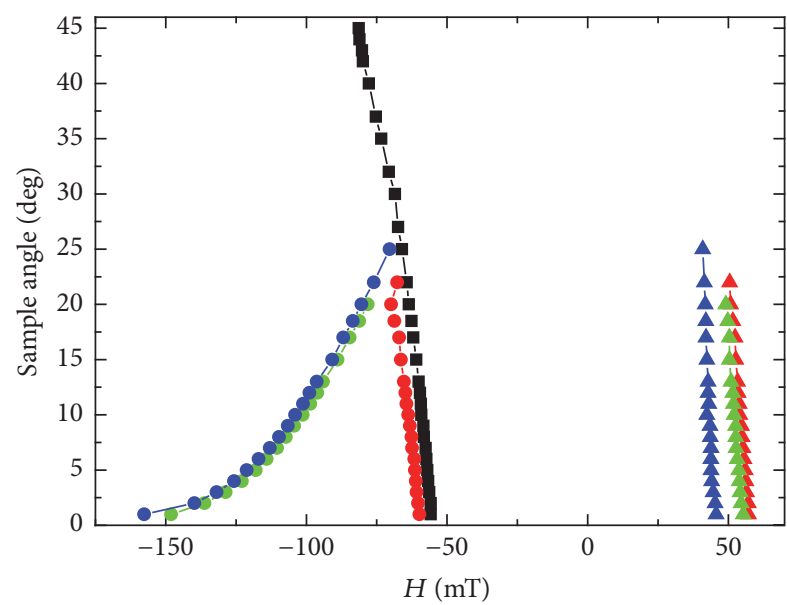

Fe with diagonal corner cuts $(200 \mathrm{~nm})^{2} \times 5 \mathrm{~nm}$ Epitaxial $\left(0^{\circ}\right)$

$$
\begin{aligned}
& -\square-H_{\text {Coerc }} \\
& -\bullet-H_{\text {Connect, Horseshoe 1 }} \\
& -\boldsymbol{-}-H_{\text {Minor, Horseshoe 1 }} \\
& -\bullet-H_{\text {Connect, Onion }}
\end{aligned}
$$

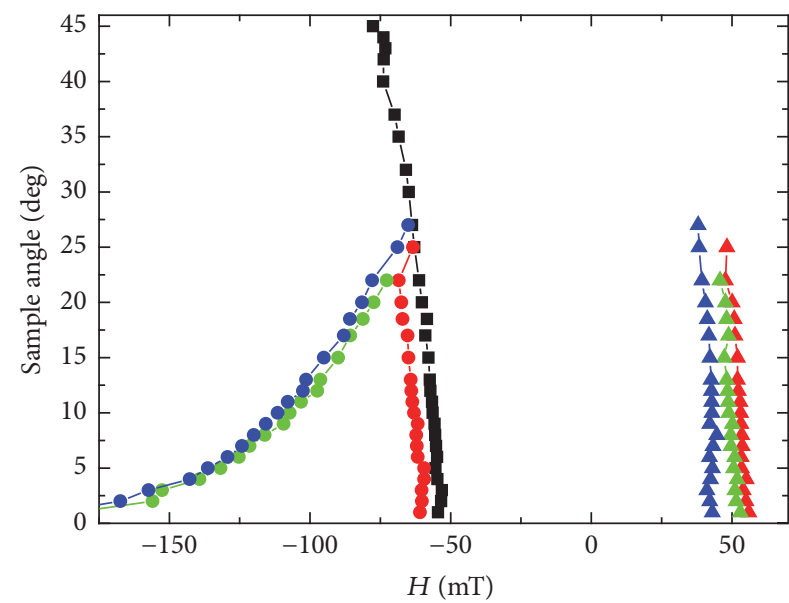

Fe with diagonal corner cuts $(200 \mathrm{~nm})^{2} \times 5 \mathrm{~nm}$ Sputtered

$$
\begin{array}{ll}
-\square-H_{\text {Coerc }} & -\mathbf{-}-H_{\text {Minor, Onion }} \\
-\bullet-H_{\text {Connect, Horseshoe 1 }} & -\bullet-H_{\text {Connect, Horseshoe 2 }} \\
-\mathbf{-}-H_{\text {Minor, Horseshoe 1 }} & -\mathbf{-}-H_{\text {Minor, Horseshoe 2 }} \\
-\bullet-H_{\text {Connect, Onion }} &
\end{array}
$$

(b)

(a)
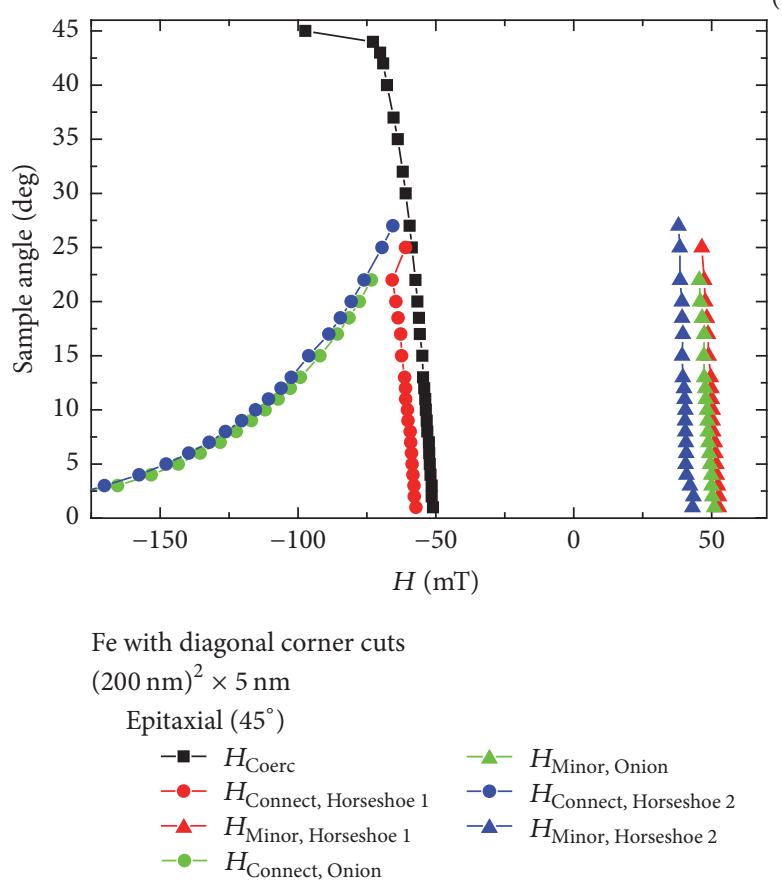

(c)

Figure 7: Phase diagrams simulated for nanorings with diagonal corner cuts, assuming epitaxial anisotropy orientation along $0^{\circ}$ (a), $45^{\circ}$ (c), and random anisotropy axes (b). The colors of the texts correspond to those in Figure 5; dot colors are identical with those in Figure 6.

via a stable intermediate onion state which can be used for technological purposes, for example, in storage devices or MRAM applications.

\section{Conflicts of Interest}

The authors declare that there are no conflicts of interest regarding the publication of this paper.

\section{References}

[1] S. A. Wolf, D. D. Awschalom, R. A. Buhrman et al., "Spintronics: a spin-based electronics vision for the future," Science, vol. 294, no. 5546, pp. 1488-1495, 2001.

[2] A. Moser, K. Takano, D. T. Margulies et al., "Magnetic recording: advancing into the future," Journal of Physics D: Applied Physics, vol. 35, no. 19, pp. R157-R167, 2002. 
[3] B. D. Terris and T. Thomson, "Nanofabricated and selfassembled magnetic structures as data storage media," Journal of Physics D: Applied Physics, vol. 38, no. 12, pp. R199-R222, 2005.

[4] J. Åkerman, “Toward a universal memory," Science, vol. 308, no. 5721, pp. 508-510, 2005.

[5] S. D. Bader, "Colloquium: Opportunities in nanomagnetism," Reviews of Modern Physics, vol. 78, no. 1, 2006.

[6] C. Chappert, A. Fert, and F. N. van Dau, "The emergence of spin electronics in data storage," Nature Materials, vol. 6, no. 11, pp. 813-823, 2007.

[7] J. Nogués, J. Sort, V. Langlais et al., "Exchange bias in nanostructures," Physics Reports, vol. 422, no. 3, pp. 65-117, 2005.

[8] W. K. Hiebert, A. Stankiewicz, and M. R. Freeman, "Direct Observation of Magnetic Relaxation in a Small Permalloy Disk by Time-Resolved Scanning Kerr Microscopy," Physical Review Letters, vol. 79, no. 6, pp. 1134-1137, 1997.

[9] R. P. Cowburn, D. K. Koltsov, A. O. Adeyeye, M. E. Welland, and D. M. Tricker, "Single-domain circular nanomagnets," Physical Review Letters, vol. 83, no. 5, pp. 1042-1045, 1999.

[10] J.-G. Zhu, Y. Zheng, and G. A. Prinz, "Ultrahigh density vertical magnetoresistive random access memory (invited)," Journal of Applied Physics, vol. 87, no. 9, pp. 6668-6673, 2000.

[11] F. Q. Zhu, D. Fan, X. Zhu, J.-G. Zhu, R. C. Cammarata, and C.L. Chien, "Ultrahigh-density arrays of ferromagnetic nanorings on macroscopic areas," Advanced Materials, vol. 16, no. 23-24, pp. 2155-2159, 2004.

[12] W. Zhang and S. Haas, "Construction of a chiral metamaterial with a U-shaped resonator assembly," Physical Review B, vol. 81, Article ID 064433, 2010.

[13] K. He, D. J. Smith, and M. R. McCartney, "Effects of vortex chirality and shape anisotropy on magnetization reversal of Co nanorings (invited)," Journal of Applied Physics, vol. 107, no. 9, p. 09D307, 2010.

[14] T. Blachowicz, A. Ehrmann Ńe Tillmanns, P. Steblinski, and L. Pawela, "Magnetization reversal in magnetic half-balls influenced by shape perturbations," Journal of Applied Physics, vol. 108, no. 12, Article ID 123906, 2010.

[15] T. Blachowicz and A. Ehrmann, "Micromagnetic simulations of anisotropies in coupled and uncoupled ferromagnetic nanowire systems," The Scientific World Journal, vol. 2013, Article ID 472597, 2013.

[16] T. Blachowicz and A. Ehrmann, "Fourfold nanosystems for quaternary storage devices," Journal of Applied Physics, vol. 110, no. 7, p. 073911, 2011.

[17] A. Ehrmann, T. Blachowicz, S. Komraus et al., "Magnetic properties of square Py nanowires: irradiation dose and geometry dependence," Journal of Applied Physics, vol. 117, no. 17, Article ID 173903, 2015.

[18] A. Ehrmann, S. Komraus, T. Blachowicz et al., "Pseudo exchange bias due to rotational anisotropy," Journal of Magnetism and Magnetic Materials, vol. 412, pp. 7-10, 2016.

[19] T. Blachowicz, A. Tillmanns, M. Fraune, R. Ghadimi, B. Beschoten, and G. Güntherodt, "Exchange bias in epitaxial CoO Co bilayers with different crystallographic symmetries," Physical Review B: Condensed Matter and Materials Physics, vol. 75, no. 5, Article ID 054425, 2007.

[20] A. Regtmeier, J. Meyer, N. Mill et al., "Influence of nanoparticular impurities on the magnetic anisotropy of self-assembled magnetic Co-nanoparticles," Journal of Magnetism and Magnetic Materials, vol. 326, pp. 112-115, 2013.
[21] M. J. Donahue and D. G. Porter, “OOMMF user's guide, version 1.0," National Institute of Standards and Technology NIST IR 6376, 1999.

[22] T. L. Gilbert, "A phenomenological theory of damping in ferromagnetic materials," IEEE Transactions on Magnetics, vol. 40, no. 6, pp. 3443-3449, 2004.

[23] A. Ehrmann and T. Blachowicz, "Influence of shape and dimension on magnetic anisotropies and magnetization reversal of $\mathrm{Py}, \mathrm{Fe}$, and Co nano-objects with four-fold symmetry," AIP Advances, vol. 5, no. 9, Article ID 097109, 2015.

[24] E. F. Kneller and R. Hawig, "The exchange-spring magnet: a new material principle for permanent magnets," IEEE Transactions on Magnetics, vol. 27, no. 4, pp. 3588-3600, 1991.

[25] J. Lindner, C. Hassel, A. V. Trunova, F. M. Römer, S. Stienen, and I. Barsukov, "Magnetism of Single-Crystalline fe nanostructures," Journal of Nanoscience and Nanotechnology, vol. 10, no. 9, pp. 6161-6167, 2010. 

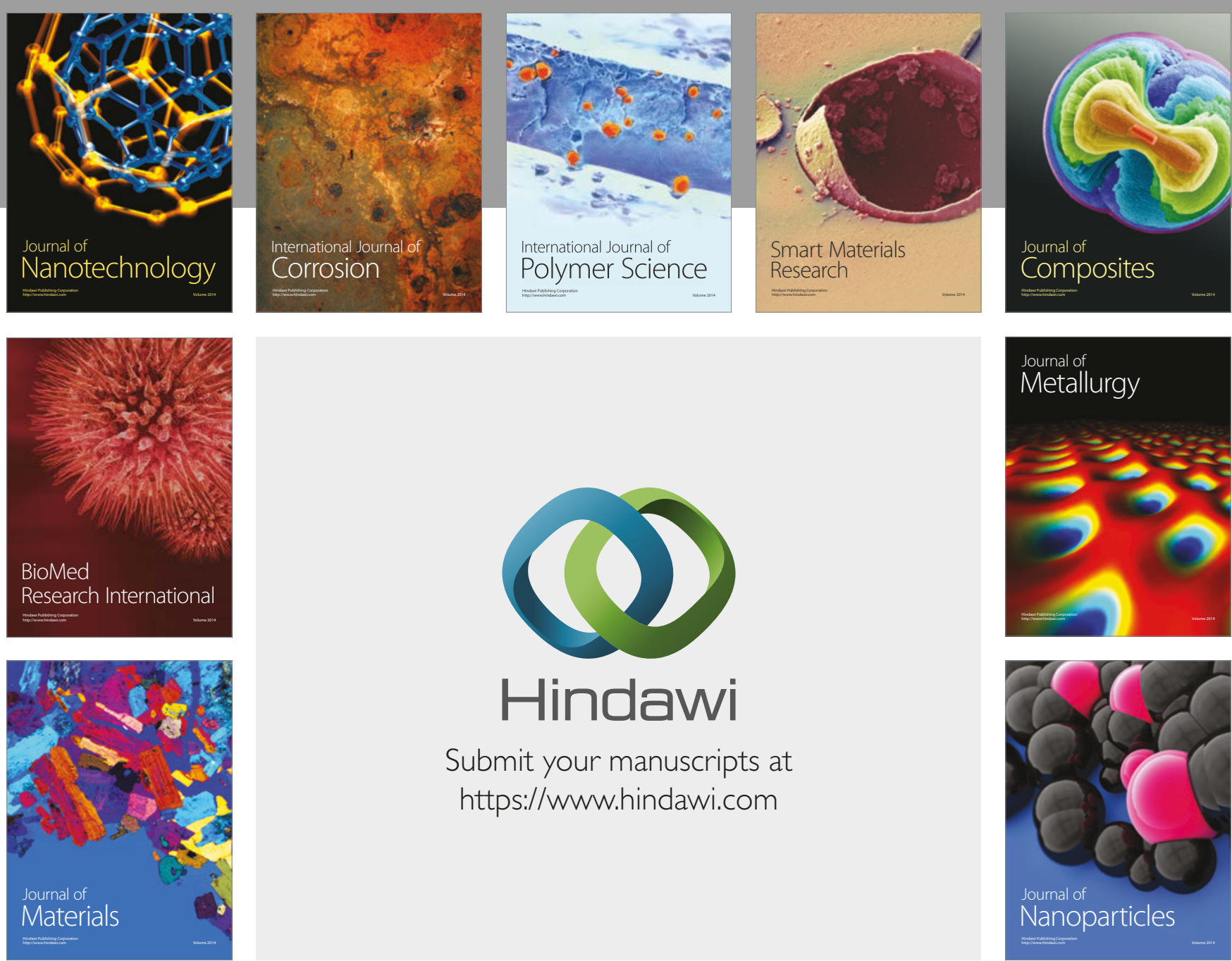

\section{Hindawi}

Submit your manuscripts at

https://www.hindawi.com
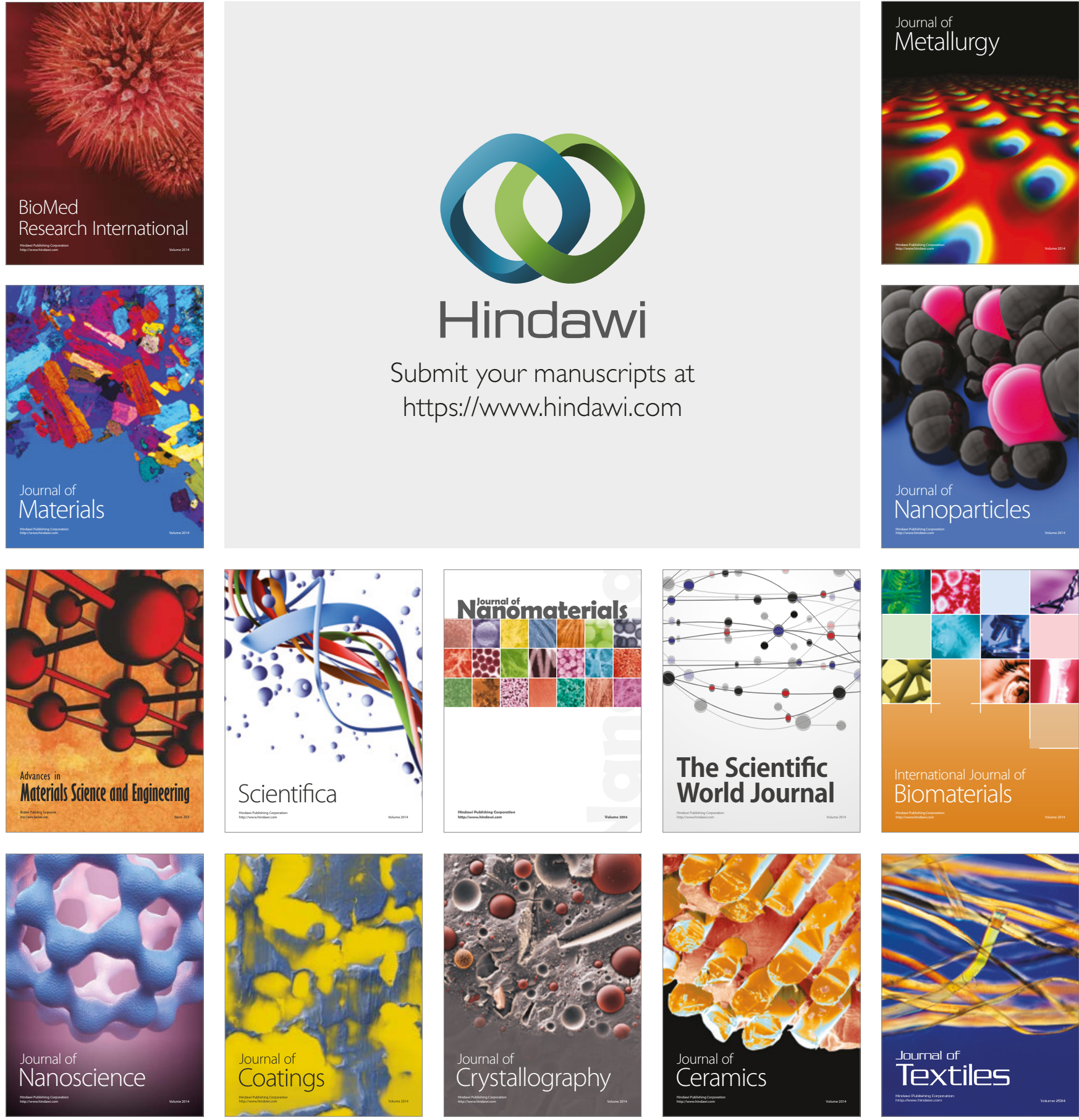

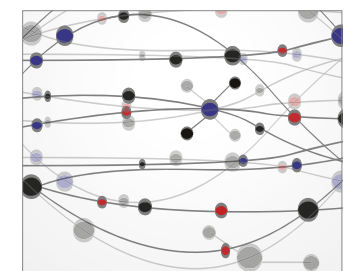

The Scientific World Journal
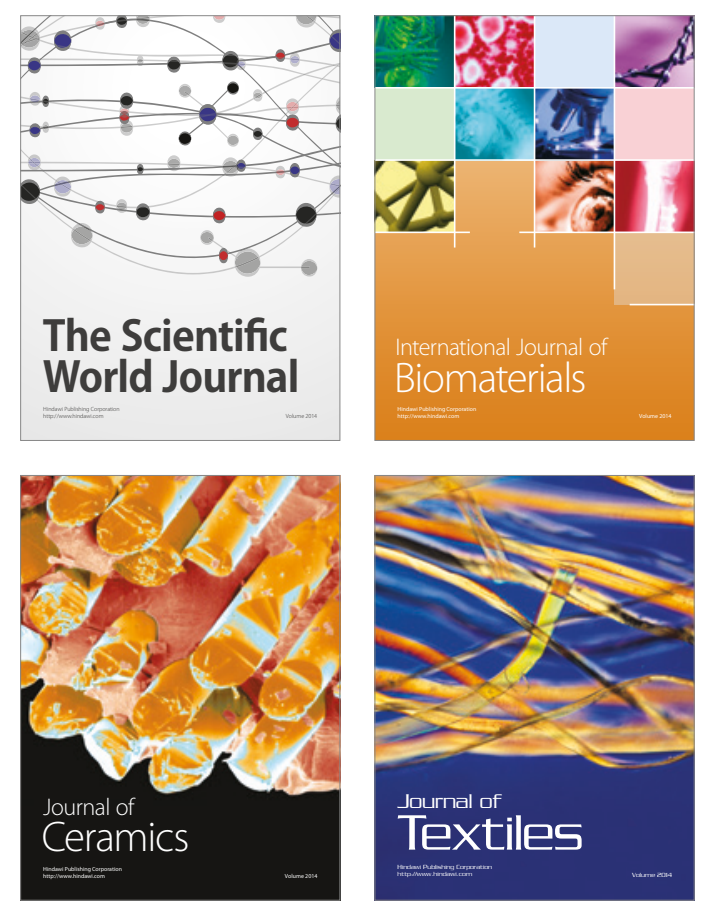